\title{
Mutagenic effects of ion beam irradiation on rice
}

\author{
Hiroyasu Yamaguchi*1,3), Yoshihiro Hase'2), Atsushi Tanaka'), Naoya Shikazono ${ }^{2,4)}$, Konosuke Degi ${ }^{1,5)}$, \\ Akemi Shimizu ${ }^{1)}$ and Toshikazu Morishita ${ }^{1,6}$ \\ 1) Institute of Radiation Breeding, National Institute of Agrobiological Sciences, 2425 Kamimurata, Hitachi-omiya, Ibaraki 319-2293, \\ Japan \\ 2) Radiation-Applied Biology Division, Japan Atomic Energy Agency, 1233 Watanuki-machi, Takasaki, Gunma 370-1292, Japan \\ 3) Present address: National Institute of Floricultural Science, 2-1 Fujimoto, Tsukuba, Ibaraki 305-8519, Japan \\ 4) Present address: Advanced Science Research Center, Japan Atomic Energy Agency, 2-4 Shirakata Shirane, Tokai, Naka, Ibaraki 319- \\ 1195, Japan \\ 5) Present address: Okinawa Prefectural Agricultural Research Center, 820 Makabe, Itoman, Okinawa 901-0336, Japan \\ 6) Present address: National Agricultural Research Center for the Hokkaido Region (Memuro), Shinsei, Memuro, Hokkaido 082-0071, \\ Japan
}

We investigated the usefulness of ion beams for mutation breeding in rice (Oryza sativa L.) by comparing the efficiency (i.e., the ratio of desirable mutations to plant damage such as lethality and sterility), mutation rate, spectrum, and optimum dose to that of gamma rays. Rice seeds were irradiated with carbon ions (mean linear energy transfer $=76$ and $107 \mathrm{keV} / \mu \mathrm{m})$, helium ions $(9 \mathrm{keV} / \mu \mathrm{m})$, and gamma rays, and their survival and fertility were examined in the $\mathrm{M}_{1}$ generation. The frequency of chlorophyll mutations and their types (albina, xantha, and viridis) were examined in the $\mathrm{M}_{2}$ generation, using the $\mathrm{M}_{1}$-plant progeny method. The efficiency of ion beams either equaled or exceeded that of gamma rays. In addition, the mutation rate of ion beams was higher than that of gamma rays. Thus, ion beams appeared to efficiently induce mutants with little radiation damage. No remarkable difference was observed in the relative frequencies of each type of mutation among 3 types of ion beams and gamma rays, thus suggesting that there was no difference in the spectrum. A shoulder dose, which hardly affected survival, was sufficient to efficiently obtain mutants for both types of radiation.

Key Words: gamma rays, ion beams, mutation frequency, optimum dose, rice, spectrum.

\section{Introduction}

Mutation breeding is a useful method for crop improvement. The type of mutagenic treatment is an important factor to obtain successful results in mutation breeding. Physical mutagens, such as gamma rays and X-rays, have mainly been used to induce mutations, and many mutant varieties have been released.

Ion beams have recently attracted attention as mutagens. A characteristic feature of ion beams is their ability to deposit high energy on a target, densely and locally, as opposed to low linear energy transfer (LET) radiation such as gamma rays and X-rays (Yang and Tobias 1979, Tanaka 1999). Due to this difference, ion beams are expected to result in different mutation induction effects and induction of novel mutants that have not been previously obtained.

Mutation induction with ion beams, using various plants, has been attempted since the 1990s in Japan. Until now, it has been demonstrated in Arabidopsis that ion beams induce

Communicated by T. Nishio

Received February 19, 2009. Accepted May 8, 2009.

*Corresponding author (e-mail: yhiroya@affrc.go.jp) mutations with high frequency and show a broad mutation spectrum, and novel mutants have been obtained (Hase et al. 2000, Shikazono et al. 2003, Tanaka et al. 1997, Tanaka et al. 2002). Furthermore, various mutants have been obtained in many crops, mainly ornamental plants (Hamatani et al. 2001, Hara et al. 2003, Kanaya et al. 2008, Kazama et al. 2008, Miyazaki et al. 2002, Miyazaki et al. 2006, Nagatomi et al. 1996, Okamura et al. 2003, Yamaguchi et al. 2003). Specific flower color mutants that could not be obtained by gamma rays, could be induced using ion beams, particularly in chrysanthemum (Nagatomi et al. 1996) and carnation (Okamura et al. 2003).

Thus, the characteristics of ion beams have been gradually clarified, and ion beam irradiation has evolved as a new mutation method. However, in terms of the mutagen for mutation breeding, the characteristics of ion beams, especially in comparison to gamma rays and X-rays, and the criterion of optimum irradiation dose for practical use have not been sufficiently clarified.

To compare the mutagenic effects of different mutagens, 2 terms, effectiveness and efficiency, have been used (Konzak et al. 1965, Mikaelsen et al. 1971, Nilan et al. 1965). Effectiveness is defined as the number of mutations 
produced per unit dose, whereas efficiency is defined as the ratio of specific desirable mutagenic changes to plant damage in the $M_{1}$ generation, such as lethality and sterility. Konzak et al. (1965) suggested that the usefulness of any mutagen in plant breeding depends not only on its mutagenic effectiveness, but also on its mutagenic efficiency. In this sense, ion beams have been shown to exhibit higher effectiveness compared to gamma rays (Fujii et al. 1966, Mei et al. 1994), X-rays (Hirono et al. 1970, Yang and Tobias 1979), and electrons (Shikazono et al. 2003). However, the efficiency of ion beams has not been compared to that of gamma rays or X-rays.

In the present study, using rice (Oryza sativa L.), we investigated the efficiency and effectiveness of ion beams and the spectrum of induced mutants in comparison to gamma rays. Furthermore, we attempted to identify the optimum dose of ion beams for use in irradiation treatment.

\section{Materials and Methods}

Oryza sativa L. cv. Hitomebore was used as our experimental material.

Hulled dry seeds of rice were placed on 6-cm-diameter petri dishes, with the embryos facing the irradiation source. The samples were irradiated with $220 \mathrm{MeV}$ carbon ions (LET $107 \mathrm{keV} / \mu \mathrm{m}$ ) at doses of 10, 20, 30, 40, 50, and 60 Gy; $320 \mathrm{MeV}$ carbon ions (LET $76 \mathrm{keV} / \mu \mathrm{m})$ at doses of 20,40 , $60,80,100$, and $150 \mathrm{~Gy}$; and $100 \mathrm{MeV}$ helium ions (LET 9 $\mathrm{keV} / \mu \mathrm{m})$ at doses of 50,100, 150, 200, 250, and $300 \mathrm{~Gy}$, generated by an AVF-cyclotron (Japan Atomic Energy Agency, Takasaki, Japan). Gamma rays were applied to unhulled dry seeds of rice at doses of 100, 150, 200, 250, 300, 350,400 , and $450 \mathrm{~Gy}$, with a dose rate of $10 \mathrm{~Gy}$ per $\mathrm{h}$, in the gamma room at the Institute of Radiation Breeding (National Institute of Agrobiological Sciences, Hitachi-omiya, Japan). Irradiation treatments were repeated 4 times for 220 and 320 $\mathrm{MeV}$ carbon-ion beams, 3 times for $100 \mathrm{MeV}$ helium-ion beam and gamma rays, using 300-1000 seeds per irradiation treatment.

One hundred seeds from each irradiation treatment were sown on moistened rock wool to investigate the effects of ion beams on survival. After a 4- or 5-day incubation at approximately $30^{\circ} \mathrm{C}$, they were moved into a greenhouse. The number of seedlings that survived 3 weeks after sowing was counted, and the survival rate was expressed as the number of seedlings from irradiated seeds divided by the number of seedlings from non-irradiated seeds. The germination rate of the non-irradiated controls was greater than $93 \%$ in both hulled and unhulled seeds.

In the irradiation treatment with $220 \mathrm{MeV}$ carbon ions at doses of $10,20,30$, and $40 ; 320 \mathrm{MeV}$ carbon ions at doses of $20,40,60,80$, and $100 ; 100 \mathrm{MeV}$ helium ions at doses of 50, 100, 150, 200, and 250; and gamma rays at doses of 100 , $150,200,250$, and 300, surviving seedlings ( $\mathrm{M}_{1}$ plant $)$ were cultured in a paddy field, and the panicles $\left(\mathrm{M}_{2}\right.$ seeds $)$ of the longest culm in each $M_{1}$ plant were harvested. Fertility and mutation were investigated using lots, which consisted of more than $150 \mathrm{M}_{1}$ plants derived from $\mathrm{M}_{1}$ seeds irradiated with each dose on the same day, and the fertility and mutation frequency were calculated for each lot.

Fertility was determined based on seed set in the panicles of $50 \mathrm{M}_{1}$ plants selected at random from each lot.

Chlorophyll mutation was investigated in the $\mathrm{M}_{2}$ generation, using the $\mathrm{M}_{1}$-plant progeny method; twenty-five $\mathrm{M}_{2}$ seeds from each $\mathrm{M}_{1}$ plant were sown individually. After germination, the number of chlorophyll mutants and germinated seedlings, and their type [albina (white), xantha (yellow), viridis (light green or yellow-green), and others such as striata (longitudinal white or yellow stripes) and maculata (green or yellow spots distributed over the leaf)] was investigated.

The mutation frequency per $\mathrm{M}_{2}$ plant shown in Fig. 3, Fig. 4 and Fig. 5 was determined as the number of chlorophyll mutants divided by the number of investigated $M_{2}$ plants. The mutation frequency per $\mathrm{M}_{1}$ plant shown as supplemental data in ESMs 1-2 was determined as the number of $\mathrm{M}_{1}$ plants that produced chlorophyll mutants in their progeny $\left(M_{2}\right.$ plant $)$ divided by the number of investigated $M_{1}$ plants. The number of mutated $M_{1}$ plants per sown $M_{1}$ seed shown in Fig. 6 was determined as the number of $M_{1}$ plants that produced chlorophyll mutants in their progeny $\left(\mathrm{M}_{2}\right.$ plant) divided by the number of sown $\mathrm{M}_{1}$ seeds. The frequency of spontaneous mutation was estimated using approximately 49000 non-irradiated plants.

The segregation frequency was investigated using strains of which 16-25 $\mathrm{M}_{2}$ seedlings germinated, and they were calculated as the number of chlorophyll mutants divided by the number of germinated $\mathrm{M}_{2}$ plants in each strain.

\section{Results}

\section{Survival}

In both ion beams and gamma rays, the dose-response curves had a shoulder at a certain dose, and survival rates rapidly decreased with doses higher than that corresponding to the shoulder (Fig. 1). The dose that corresponds to the shoulder was $20 \mathrm{~Gy}$ with the $220 \mathrm{MeV}$ carbon-ion beam, 60 $80 \mathrm{~Gy}$ with the $320 \mathrm{MeV}$ carbon-ion beam, $150 \mathrm{~Gy}$ with the $100 \mathrm{MeV}$ helium-ion beam, and $200 \mathrm{~Gy}$ with gamma rays. The $50 \%$ lethal dose was $35 \mathrm{~Gy}$ with the $220 \mathrm{MeV}$ carbonion beam, $100 \mathrm{~Gy}$ with the $320 \mathrm{MeV}$ carbon-ion beam, 240 Gy with the $100 \mathrm{MeV}$ helium-ion beam, and $300 \mathrm{~Gy}$ with gamma rays. Thus, the effect on lethality increased with increasing LET.

\section{Fertility}

The fertility decreased linearly with increasing irradiation dose (Fig. 2). The effect on fertility also increased with increasing LET in the following order: $220 \mathrm{MeV}$ carbon-ion beam $<320 \mathrm{MeV}$ carbon-ion beam $<100 \mathrm{MeV}$ helium-ion beam $<$ gamma rays. 


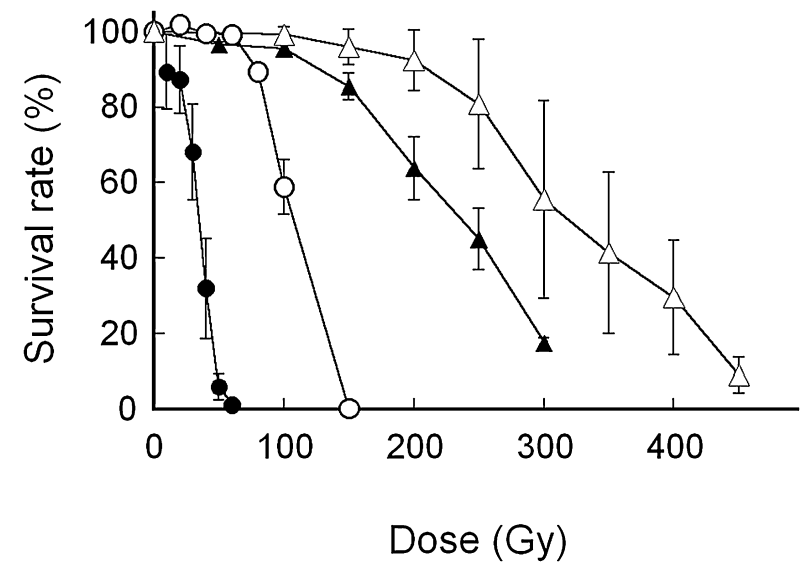

Fig. 1. Effect of ion beam and gamma ray irradiation on the survival of rice seeds. Survival rate is expressed as the number of seedlings from the irradiated seeds divided by the number of seedlings from the non-irradiated seeds. 0 : $220 \mathrm{MeV}$ carbon-ion beam; $\bigcirc: 320 \mathrm{MeV}$ carbon-ion beam; $\boldsymbol{\Delta}$ : $100 \mathrm{MeV}$ helium-ion beam; $\triangle$ : gamma rays. Vertical bars indicate SE ( $n=4$ for the 220 and $320 \mathrm{MeV}$ carbon-ion beams; $n=3$ for the $100 \mathrm{MeV}$ helium-ion beam and gamma rays).

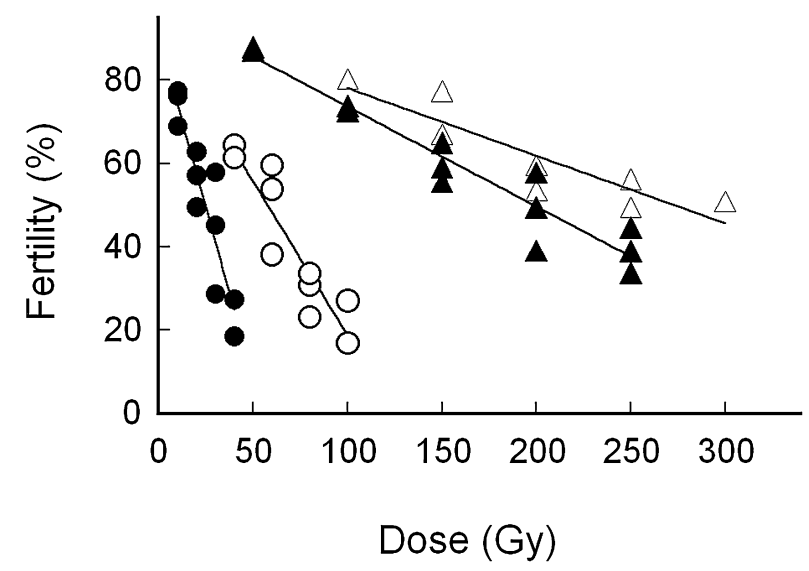

Fig. 2. Effect of ion beam and gamma ray irradiation on fertility. Fertility is determined based on seed set in the panicles of $50 \mathrm{M}_{1}$ plants selected at random from each lot, which consisted of more than 150 $\mathrm{M}_{1}$ plants derived from $\mathrm{M}_{1}$ seeds irradiated with each dose on the same day. $: 220 \mathrm{MeV}$ carbon-ion beam, $y=90.6-1.64 x, r=-0.918^{* * *} ; \bigcirc$ : $320 \mathrm{MeV}$ carbon-ion beam, $y=92.6-0.738 x, r=-0.913^{* * *} ; \mathbf{\Delta}: 100$ $\mathrm{MeV}$ helium-ion beam, $y=97.6-0.240 x, r=-0.959^{* * *} ; \triangle$ : gamma rays, $y=94.2-0.162 x, r=-0.896 * *{ }^{* *}$ and $* * *$ Significant at $1 \%$ and $0.1 \%$ levels, respectively.

\section{Effectiveness}

To evaluate the "effectiveness", the relationship between the irradiation dose and the mutation frequency per $\mathrm{M}_{2}$ plant is shown in Fig. 3. The frequency of chlorophyll mutation increased linearly with increasing irradiation dose. The dose required to obtain the same mutation frequency increased in the following order: $220 \mathrm{MeV}$ carbon-ion beam $<320 \mathrm{MeV}$ carbon-ion beam $<100 \mathrm{MeV}$ helium-ion beam < gamma rays. Thus, the effectiveness also increased with LET, indicating that the "effectiveness" of ion beams was higher than that of gamma rays. The frequency of spontaneous mutation was approximately $0.02 \%$.

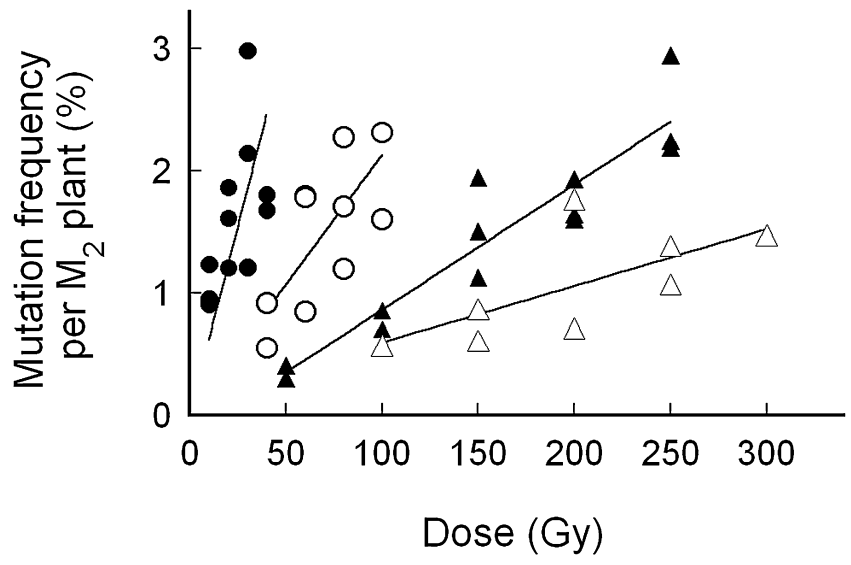

Fig. 3. Effect of ion beam and gamma ray irradiation on mutation induction. The mutation frequency is determined as the number of chlorophyll mutants divided by the number of $\mathrm{M}_{2}$ plants investigated, using the $\mathrm{M}_{1}$-plant progeny method. $0: 220 \mathrm{MeV}$ carbon-ion beam, $y=$ $0.876+0.0305 x, r=0.563 ; \bigcirc: 320 \mathrm{MeV}$ carbon-ion beam, $y=0.145+$ $0.0193 x, r=0.696 ; \boldsymbol{\Delta}: 100 \mathrm{MeV}$ helium-ion beam, $y=-0.171+$ $0.0103 x, r=0.934 * * ;:$ gamma rays, $y=0.124+0.00464 x, r=0.685$. ** Significant at $1 \%$ level.

\section{Eefficiency}

To evaluate the "efficiency" on the basis of lethality, the relationship between the mutation frequency and the survival rate is shown in Fig. 4 and ESM 1. Even if the same radiation type was applied at the same dose, the survival rate and the mutation frequency differed among irradiation treatments, as shown in Fig. 1 and Fig. 3, respectively. Therefore, the mutation frequency and the survival rate of each irradiation treatment were plotted, and the relation between them is shown.

The mutation frequency per $\mathrm{M}_{2}$ plant increased inversely with the survival rate following the irradiation treatment (Fig. 4). The relationship between the mutation frequency per $\mathrm{M}_{2}$ plant and survival rate was not linear; in the range where the survival rates were $90-100 \%$, the mutation frequencies increased markedly. In contrast, in the range where the survival rates were $90 \%$ or less, the mutation frequency increased gradually. Therefore, the mutation frequencies were compared at $70 \%$ and $90 \%$ survival rates. At a $90 \%$ survival rate, the mutation frequency per $\mathrm{M}_{2}$ plant with the 3 types of ion beams was approximately $1.7 \%$, whereas that of gamma rays was lower than those of the ion beams, approximately $1.0 \%$. At $70 \%$ survival rate, the $320 \mathrm{MeV}$ carbon-ion beam showed the highest mutation frequency $(2.0 \%)$, followed by the $100 \mathrm{MeV}$ helium-ion beam $(1.9 \%), 220 \mathrm{MeV}$ carbon-ion beam $(1.8 \%)$, and gamma rays $(1.3 \%)$.

The relationship between the mutation frequency per $M_{1}$ plant and the survival rate was similar to the case of the mutation frequency per $\mathrm{M}_{2}$ plant with each radiation (ESM 1). At a $90 \%$ survival rate, the $320 \mathrm{MeV}$ carbon-ion beam showed the highest mutation frequency $(9.5 \%)$, followed by the $220 \mathrm{MeV}$ carbon-ion beam $(9.3 \%)$, gamma rays $(8.4 \%)$, and $100 \mathrm{MeV}$ helium-ion beam (8.1\%). At a $70 \%$ survival 




\section{Survival rate $(\%)$}

Fig. 4. Relationship between survival rate and mutation frequency. The mutation frequency is determined as the number of chlorophyll mutants divided by the number of $\mathrm{M}_{2}$ plants investigated, using the $\mathrm{M}_{1}$-plant progeny method. Survival rate is expressed as the number of seedlings from irradiated seeds divided by the number of seedlings from the non-irradiated seeds. The regression lines are calculated in the range of $60 \%-95 \%$ survival and $90 \%-105 \%$ survival individually. $220 \mathrm{MeV}$ carbon-ion beam, $y=1.77-0.0001 x, r=-0.005$ (for $60 \%-$ $95 \%$ survival) and $y=6.91-0.057 x, r=-0.496$ (for $90 \%-105 \%$ survival); $320 \mathrm{MeV}$ carbon-ion beam, $y=2.70-0.011 x, r=-0.280$ (for $60 \%-95 \%$ survival) and $y=10.2-0.093 x, r=-0.492$ (for $90 \%-105 \%$ survival); $100 \mathrm{MeV}$ helium-ion beam, $y=3.1-0.018 x, r=-0.635^{*}$ (for $60 \%-95 \%$ survival) and $y=15.8-0.156 x, r=-0.614$ (for $90 \%-$ $105 \%$ survival); gamma rays, $y=2.18-0.013 x, r=-0.445$ (for $60 \%$ $95 \%$ survival) and $y=8.36-0.074 x, r=-0.825$ (for $90 \%-105 \%$ survival). * Significant at $5 \%$ level.

rate, the $100 \mathrm{MeV}$ helium-ion beam showed the highest mutation frequency $(10.2 \%)$, followed by the $220 \mathrm{MeV}$ carbon-ion beam $(9.3 \%), 320 \mathrm{MeV}$ carbon-ion beam $(8.1 \%)$, and gamma rays $(6.6 \%)$.

Thus, it was suggested that the "efficiency" on the basis of lethality of ion beams was higher than that of gamma rays. The difference of "efficiency" on the basis of lethality between ion beams and gamma rays was clear in the mutation frequency per $\mathrm{M}_{2}$ plant in comparison with the mutation frequency per $\mathrm{M}_{1}$ plant.

To evaluate the "efficiency" on the basis of fertility, the relationship between the mutation frequency and the fertility is shown in Fig. 5 and ESM 2. The fertility was also differed among irradiation treatments even if the same radiation type was applied at the same dose, as shown in Fig. 2. Therefore, the mutation frequency and the fertility of each irradiation treatment were plotted, and their relationship is shown. The fertility and the mutation frequency per $\mathrm{M}_{2}$ plant showed a negative linear relationship for each type of radiation. The mutation frequency of ion beams increased significantly with decreasing fertility, and the same tendency was observed for gamma rays. The mutation frequencies at $60 \%$ fertility of the $220 \mathrm{MeV}$ carbon-ion beam and the $100 \mathrm{MeV}$ helium-ion beam were $1.4 \%$, whereas those of $320 \mathrm{MeV}$ carbon-ion beam and gamma rays were lower, i.e., 1.1\%.

The fertility and the mutation frequency per $\mathrm{M}_{1}$ plant also showed a negative linear relationship (ESM 2). The mutation frequency increased significantly and inversely with fertility in $220 \mathrm{MeV}$ carbon-ion beam, the $100 \mathrm{MeV}$ heliumion beam and gamma rays. At $60 \%$ fertility, the $100 \mathrm{MeV}$ helium-ion beam showed the highest mutation frequency $(8.0 \%)$, followed by the $220 \mathrm{MeV}$ carbon-ion beam $(7.5 \%)$, $320 \mathrm{MeV}$ carbon-ion beam (7.2\%), and gamma rays $(6.7 \%)$.

Thus, on the basis of fertility, ion beams induced higher frequencies of mutation than gamma rays, suggesting that the "efficiency" on the basis of fertility of ion beams was higher than that of gamma rays. Also in the efficiency on the basis of fertility, the difference between ion beams and gamma rays was clear in the mutation frequency per $\mathrm{M}_{2}$ plant in comparison with the mutation frequency per $\mathrm{M}_{1}$ plant.

\section{Optimum irradiation dose}

The optimum irradiation dose for obtaining the highest number of mutants from the irradiated seeds was clarified from the relationship between the irradiation dose and the number of mutated $M_{1}$ plants per sown $M_{1}$ seed (Fig. 6). In both ion beams and gamma rays, the number of mutated $M_{1}$ plants per sown $\mathrm{M}_{1}$ seed reached a maximum at a certain dose; at higher doses, the number of mutated $\mathrm{M}_{1}$ plants per sown $\mathrm{M}_{1}$ seed decreased with increasing dose. When quadratic curves were fitted to the plots for each radiation, the maximum numbers of mutated $M_{1}$ plants per sown $M_{1}$ seed was 0.085 at $22 \mathrm{~Gy}$ with the $220 \mathrm{MeV}$ carbon-ion beam, 0.094 at $73 \mathrm{~Gy}$ with the $320 \mathrm{MeV}$ carbon-ion beam, 0.076 at $187 \mathrm{~Gy}$ with the $100 \mathrm{MeV}$ helium-ion beam, and 0.058 at $209 \mathrm{~Gy}$ with gamma rays. Thus, the maximum numbers of mutated $M_{1}$ plants per sown $M_{1}$ seed of the 3 types of ion beams were higher than that of gamma rays. The dose at which the number of mutated $M_{1}$ plants per sown $M_{1}$ seed 


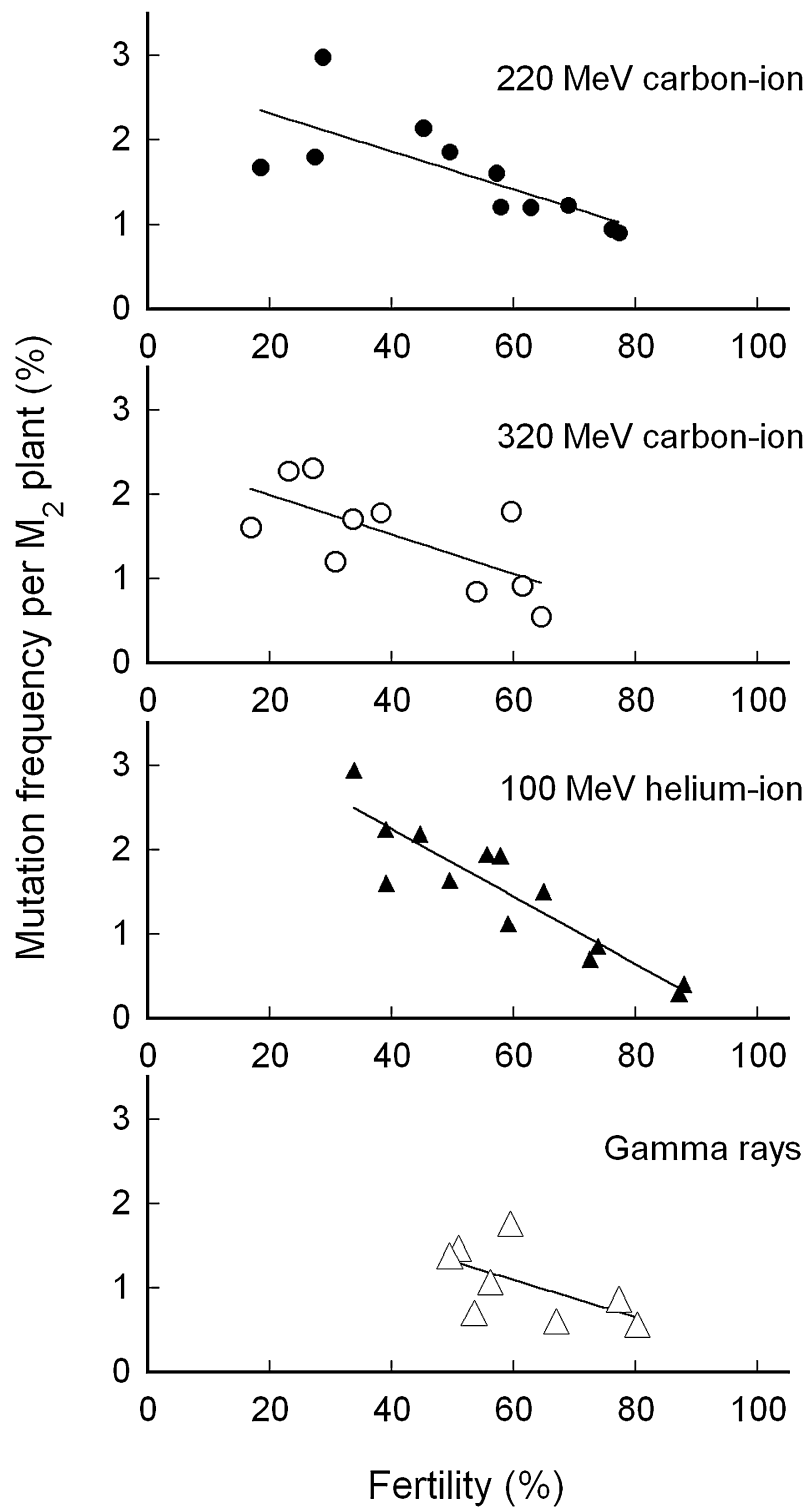

Fig. 5. Relationship between fertility and mutation frequency. The mutation frequency is determined as the number of chlorophyll mutants divided by the number of $\mathrm{M}_{2}$ plants investigated, using the $\mathrm{M}_{1^{-}}$ plant progeny method. Fertility is based on seed set in panicles of the longest culm in $50 \mathrm{M}_{1}$ plants selected at random in each treatment. $220 \mathrm{MeV}$ carbon-ion beam, $y=2.76-0.0225 x, r=-0.743^{* *} ; 320 \mathrm{MeV}$ carbon-ion beam, $y=3.23-0.0383 x, r=-0.644 * ; 100 \mathrm{MeV}$ helium-ion beam, $y=3.84-0.0400 x, r=-0.911^{* *}$; gamma rays, $y=2.41-0.022 x$, $r=-0.587$. $^{*}$ and $* *$ Significant at 5 and $1 \%$ levels, respectively.

was highest almost corresponded to the shoulder appearing in the survival curves for both ion beams and gamma rays (Fig. 1).

\section{Spectrum of chlorophyll mutation}

Both ion beams and gamma rays induced albina, xantha, viridis, and other mutants such as striata (longitudinal white or yellow stripes) and maculata (green or yellow spots distributed over the leaf). Regardless of radiation type and irradiation dose, the frequency of albina was highest, followed

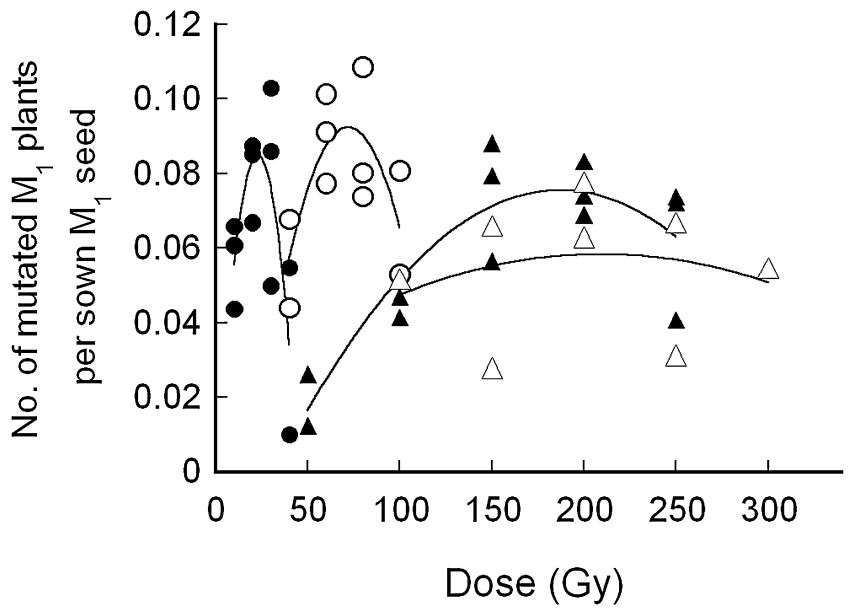

Fig. 6. Relationship between irradiation dose and the number of mutated $M_{1}$ plants per sown $M_{1}$ seed. The number of mutated $M_{1}$ plants per sown $M_{1}$ seed is determined as the number of $M_{1}$ plants that produced chlorophyll mutants in their progeny $\left(\mathrm{M}_{2}\right.$ plant) divided by the number of sown $\mathrm{M}_{1}$ seeds sown after irradiation. $0: 220 \mathrm{MeV}$ carbonion beam, $y=-0.696+0.780 x-0.0174 x^{2}, r=0.732 * ; \bigcirc: 320 \mathrm{MeV}$ carbon-ion beam, $y=-8.52+0.493 x-0.0034 x^{2}, r=0.726^{*} ; \boldsymbol{\Delta}: 100 \mathrm{MeV}$ helium-ion beam, $y=-3.462+0.118 x-0.000315 x^{2}, r=0.869 * * * ; \triangle$ : gamma rays, $y=1.83+0.0383 x-0.0000916 x^{2}, r=0.218 . *$ and $* * *$ Significant at $5 \%$ and $0.1 \%$ levels, respectively.

by viridis (Fig. 7). When totaled at each radiation type, the frequency of albina was about $50 \%$, whereas that of xantha ranged from $8 \%-15 \%$, that of viridis ranged from $25 \%-$ $30 \%$, and that of other mutations ranged from $8 \%-13 \%$ in each radiation type. Thus, no remarkable difference in the relative frequencies of each type of mutation was observed among the 3 types of ion beams and gamma rays.

\section{Segregation frequency}

In each radiation type, the strain that showed high segregation frequency increased with irradiation of a higher dose. As a result, the average of the segregation frequency increased. The segregation frequencies produced by ion beams seemed to be higher than those produced by gamma rays; the segregation frequencies for chlorophyll mutants induced by gamma rays did not increase above 0.17 when the dose increased from 200 Gy to $300 \mathrm{~Gy}$ (Fig. 8). In contrast, segregation frequencies for mutants induced by $220 \mathrm{MeV}$ carbonion beam were $0.19-0.20$ in the range of $20-40 \mathrm{~Gy}$, those by $320 \mathrm{MeV}$ carbon-ion beam were $0.18-0.22$ in the range of $60-100 \mathrm{~Gy}$, and those by $100 \mathrm{MeV}$ helium-ion beam were $0.16-0.20$ in the range of $100-250 \mathrm{~Gy}$.

\section{Discussion}

On the basis of fertility, the mutation frequency induced by ion beams equaled or exceeded that induced by gamma rays. On the basis of lethality, ion beams induced higher frequencies of mutation than gamma rays. Furthermore, the maximum number of mutated $\mathrm{M}_{1}$ plants per $\mathrm{M}_{1}$ seed in 3 types of 

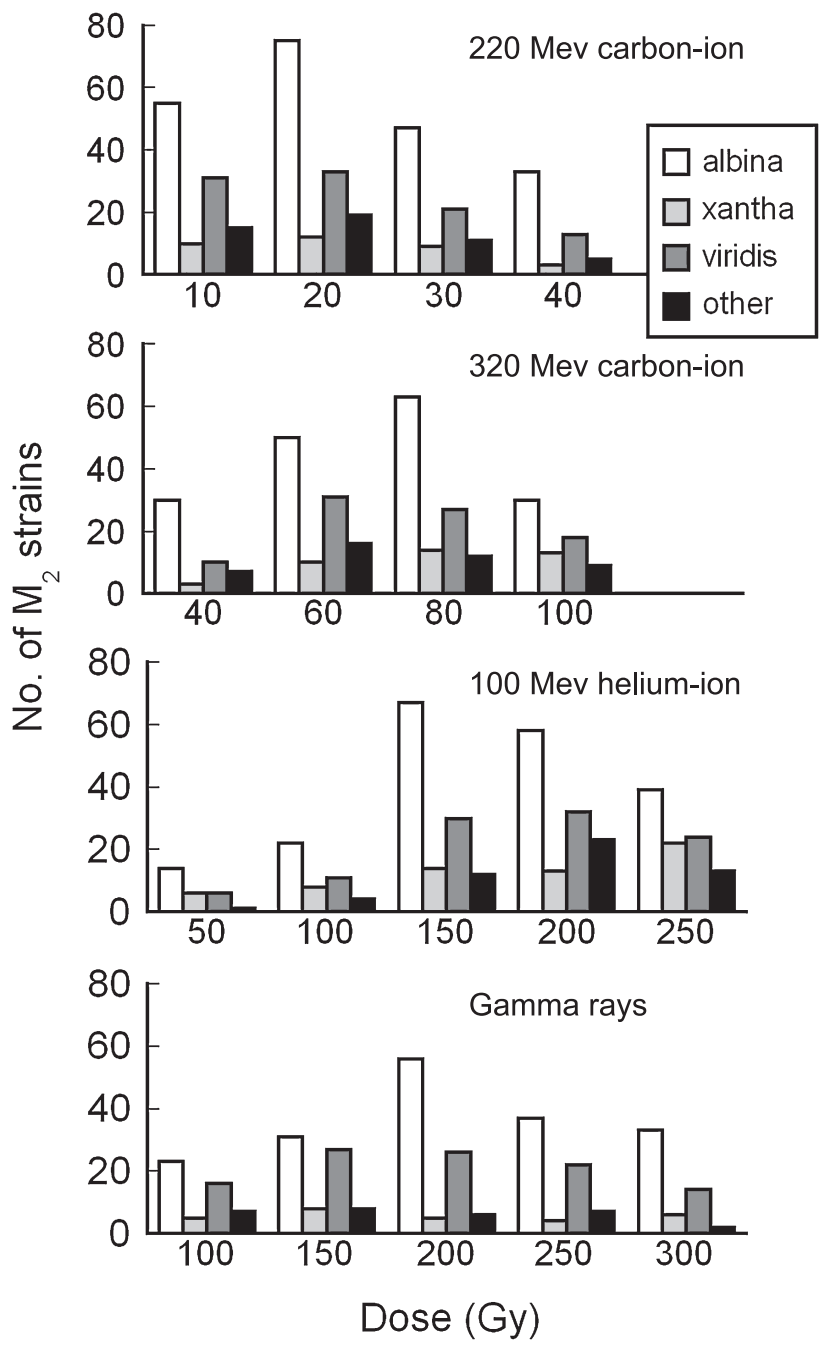

Fig. 7. Frequency distribution of the different types of chlorophyll mutants induced by ion beam and gamma ray irradiation.

ion beams were higher than that in gamma rays. Both in ion beams and gamma rays, number of mutated $\mathrm{M}_{1}$ plants per sown $\mathrm{M}_{1}$ seed reached a maximum at the shoulder dose, which did not affect survival markedly. Accordingly, this result also demonstrated that ion beams had high "efficiency" on the basis of lethality. Thus, it was suggested that the efficiency of ion beams was equal or superior to that of gamma rays.

Ekberg (1969) reported that $82 \%$ of sterility induced by ionizing radiations (neutrons and X-rays) was predominantly caused by chromosome aberrations (translocations and inversions) in barley. Furthermore, the sterility caused by recessive mutations does not appear until the $\mathrm{M}_{2}$ generation. Therefore, it was believed that the sterility induced by ion beams and gamma rays also was mainly caused by chromosome aberrations, as with neutrons and X-rays, though these factors of sterility were not clarified from our study. Consequently, it was believed that high "efficiency" on the basis of fertility means that mutation frequency is high in the degree of chromosome aberrations. Yang and Tobias (1979) sug- gested that gamma rays cause more deleterious effects to cells than high-LET radiation when a certain density of genetic lesions is required to produce a given mutation, and as a result, heavy ions could potentially induce viable mutants at a higher efficiency than gamma rays. Thus, ion beams appear to efficiently induce mutants with little radiation damage.

Some of the translocations and inversions were transmittable to the next generation (Ekberg 1969), thus causing sterility in later generations. Consequently, lower sterility in the $M_{1}$ generation is desirable for seed propagated crops. Also, in vegetative propagated crops, lower chromosome aberrations are desirable because the $\mathrm{M}_{1}$ generations are used directly as mutants. For these reasons, it was believed that a mutagen of "high efficiency" on the basis of fertility is better for obtaining a useful mutant.

On the other hand, the relationship between the mutation frequency and irradiation dose showed that irradiation with a low dose of ion beams can induce the same mutation frequency induced by gamma rays. Therefore, the mutation frequencies per unit dose were higher with ion beams than with gamma rays, thus indicating that ion beams had a higher mutation effectiveness than gamma rays, which confirms the results of previous studies (Fujii et al. 1966, Mei et al. 1994).

The difference of the mutation frequency between ion beams and gamma rays was slightly small in the mutation frequency per $\mathrm{M}_{1}$ plant in comparison with the mutation frequency per $\mathrm{M}_{2}$ plant. It was thought that it was due to the difference in the number of initial cells after irradiation between ion beams and gamma rays; the number of initial cells after ion beam irradiation was less than that after gamma ray irradiation, as discussed later. The expected mutation frequency per $\mathrm{M}_{1}$ plant differs due to the number of initial cells in the seed; mutation frequency per $\mathrm{M}_{1}$ plant rises with increasing cells. Therefore, the mutation frequency per $\mathrm{M}_{1}$ plant of gamma rays was evaluated higher than that of ion beams even if the mutation frequencies per initial cells were the same between ion beams and gamma rays. For this reason, it was thought that the mutation induction effect should be compared using the mutation frequency per $\mathrm{M}_{2}$ plant considering the number of initial cells after treatments of mutagens.

For rice, Kawai advised to harvest $\mathrm{M}_{2}$ seeds from early developed panicles in order to obtain many mutants of independent genetic origin (qtd. in van Harten 1998). Osone (1963) reported that the generative tissues of the panicles in main culm were derived from corpus initial cells of the embryo, which consists of about 5 or 6 cells in maximum. Accordingly, the panicle of the main culm is chimeric, and therefore the segregation frequency of chlorophyll mutation in $\mathrm{M}_{2}$ seeds from main culm becomes less than 0.25 (Osone 1963). In our study, we harvested the panicles $\left(M_{2}\right.$ seeds) of the longest culms in each $\mathrm{M}_{1}$ plant, probably which were main culms, and the averages of the segregation frequency with every irradiation treatment were less than 0.25 .

The number of strains with high segregation frequency increased with the irradiation doses. The segregation frequency shows the ratio of parts derived from the mutated 


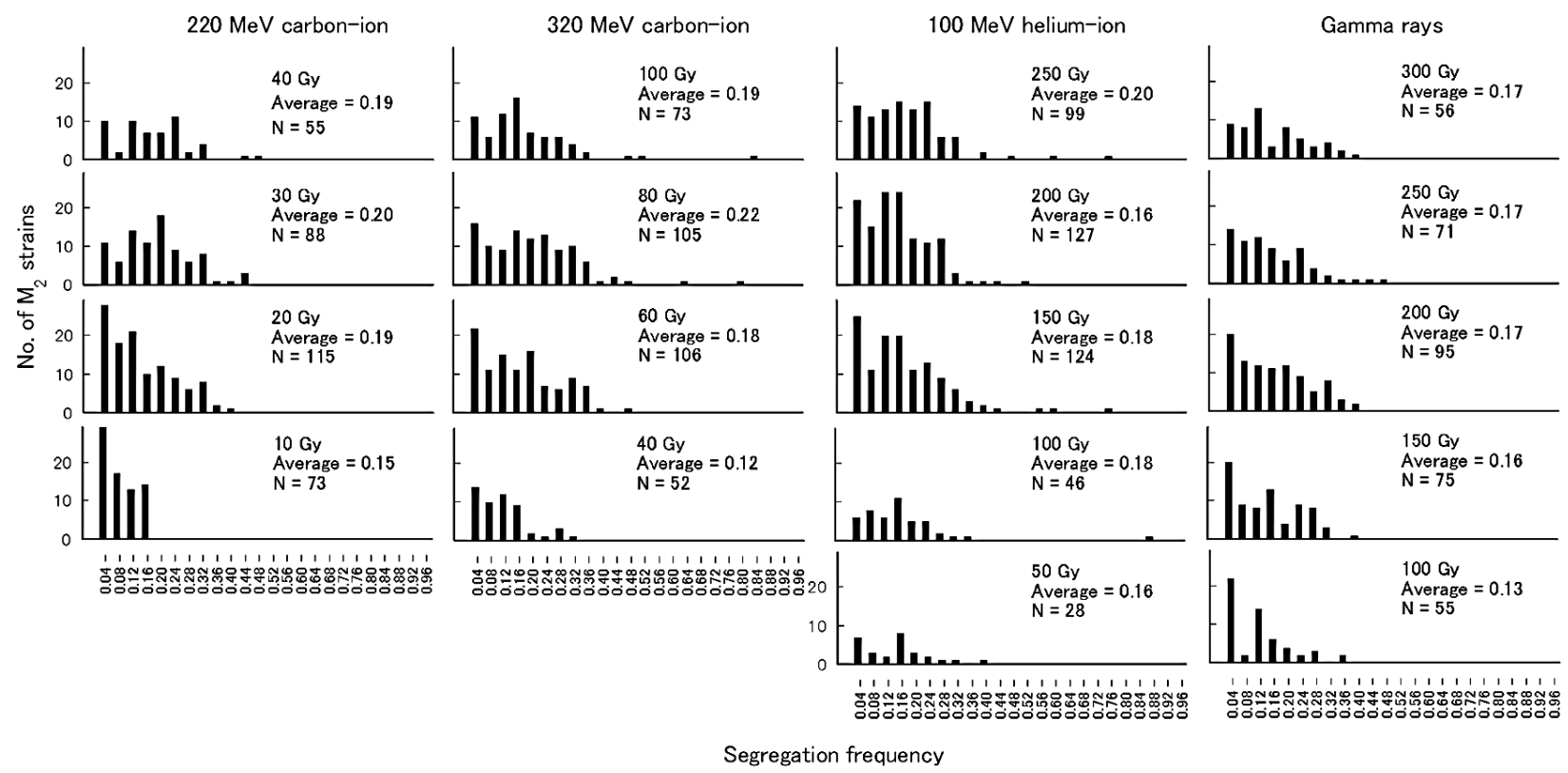

Fig. 8. Frequency distribution of segregation frequency of chlorophyll mutants induced by ion beam and gamma ray irradiation.

cell in the panicle, i.e., the width of the mutated sector. It is unlikely that the parts derived from the mutated cell broaden without a change in the number of initial cells when the irradiation dose increases. It was thought that the increase of the segregation frequency was due to the decreasing number of initial cells as previously discussed by Yamaguchi (1962) and Osone (1963).

The segregation frequencies of ion beams were higher than those of gamma rays. This result indicates that the number of initial cells in seeds after irradiation with ion beams was smaller than that with gamma rays. This difference between ion beams and gamma rays may have been due to the following reason; ion beams cause serious damage because of their high LET, and compared to the number of gamma rays, a relatively small number of ion beams penetrate through cells (Tanaka 1999). It was assumed that some initial cells died even with a low irradiation dose of ion beams, and dead cells with serious damage would be mixed with live cells with little or no damage in apical meristems irradiated with ion beams. In contrast, it was assumed that chromosomes are uniformly irradiated at many points by gamma rays (Yang and Tobias 1979), and as a result, the degree of damage would not differ greatly among initial cells; almost all initial cells were uniformly dead or alive according to the irradiation dose. Thus, it was believed that this difference between ion beams and gamma rays causes the difference in the number of initial cells.

A wide sector is also useful because it facilitates the screening and establishment of mutants in mutation breeding, and it was shown that ion beams were superior with regard to this point.

New mutagens are expected to have a different spectrum from the mutagens presently used. In the comparison of the spectrum of chlorophyll mutations, significant differences between ion beams and gamma rays were not revealed, because the relative frequency of each type of chlorophyll mutation induced by ion beams was similar to that induced by gamma rays; the frequency of albina was highest, followed by viridis. This tendency was previously observed in rice treated with gamma rays (Ando 1968, Yamaguchi 1962), Xrays (Matsuo et al. 1958), and thermal neutrons (Matsuo et al. 1958, Yamaguchi 1962). The same results were obtained using gamma rays (Doll and Sandfaer 1969), X-rays (Gustafsson 1969), and neutrons (Gustafsson 1969) in similar studies performed with barley. However, the spectrum of mutations induced in barley by 2 chemical mutagens, ethylene oxide (Gustafsson 1969) and ethyl methanesulfonate (Doll and Sandfaer 1969), differed from that induced by radiation; the frequency of viridis was higher than that of albina. The results of our study suggest that the relative frequency of each chlorophyll mutation induced by radiation did not vary among types of radiation, and it is likely to differ from the frequencies produced by chemical mutagens.

We found no difference in the spectrum of chlorophyll mutations between ion beams and gamma rays. Naito et al. (2005) showed that both high-LET carbon ions and lowLET gamma rays generate large deletions, regardless of the irradiation dose, and hypothesized that most deletions are not transmitted to progeny. In our study, the comparison of the spectrum was performed in $\mathrm{M}_{2}$ generation only on the mutations caused by deletions that were transmitted. This might be the reason why there were no differences in the spectrum between radiation type or irradiation dose. In chrysanthemum (Nagatomi et al. 1996) and carnation (Okamura et al. 2003), it has previously been reported that specific flower color mutants that could not be obtained by gamma rays, could be induced using ion beams. Naito et al. (2005) also described that non-transmissible mutations can survive 
over generations in vegetative propagated crops and have probably contributed to the genetic improvement of such crops. The differences of the spectrum between ion beams and gamma rays might have appeared because both transmissible and non-transmissible mutations can be detected in vegetative propagated crops such as chrysanthemum and carnation. However, further investigations are necessary to reveal differences in the spectrum of mutations, and it might be clarified during its use for mutation breeding aimed at various target traits in many crops.

For practical uses of ion beams, the criteria for the irradiation dose are necessary. With regard to the optimum irradiation dose, to our knowledge, there are no experiment-based reports on gamma rays. Therefore, in the present study, we examined suitable irradiation doses from the perspective of obtaining the maximum number of mutant lines from the seeds sown after irradiation in both ion beams and gamma rays. As a result, the number of mutated lines per irradiated seed was highest at a dose that corresponded to the shoulder appearing in the survival curves in both ion beams and gamma rays. Irradiation at the shoulder dose does not affect survival markedly, approximately 90\% survival. Our result demonstrated that irradiation with such low dose was enough to efficiently produce mutants. For gamma ray treatment, a growth reduction for $\mathrm{M}_{1}$ seedlings of $30 \%-50 \%$ or a survival rate of $40 \%-60 \%$ in control plants has often been considered the criterion for a promising treatment. In order to avoid considerable change in the genetic background (van Harten 1998), treatments are nowadays performed with lower doses than the doses used for those criteria. The shoulder dose is lower in comparison to the $50 \%$ lethal dose, which has been considered a criterion for a promising treatment in gamma rays. Therefore, the change of the genetic background appeared to be lower, but this is outside the scope of our study. Consequently, the shoulder dose is considered to be a more suitable criterion for the assessment of irradiation treatment for efficiently obtaining useful mutants without considerably changing their genetic background.

\section{Acknowledgments}

We would like to thank Dr. Yasuo Ukai for the valuable discussions. Part of this study was financially supported by the Budget for Nuclear Research of the Ministry of Education, Culture, Sports, Science, and Technology, based on the screening and counseling by the Atomic Energy Commission.

\section{Literature Cited}

Ando,A. (1968) Mutation induction in rice by radiation combined with chemical protectants and mutagens. In: Rice breeding with induced mutations. Report of an FAO/IAEA research co-ordination meeting on the use in induced mutations in rice breeding, held in Taipei, Republic of China, 5-9 June 1967, International Atomic Energy Agency, Vienna, pp. 7-15.

Doll,H. and J.Sandfaer (1969) Mutagenic effect of gamma rays, diethyl sulphate, ethyl methanesulphonate, and various combinations of gamma rays and the chemicals. In: Induced mutations in plants. Proceedings of a symposium on the nature, induction and utilization of mutations in plants jointly organized by the IAEA and FAO and held at Pullman, Wash., 14-18 July 1969, International Atomic Energy Agency, Vienna, pp. 195-206.

Ekberg, I. (1969) Different types of sterility induced in barley by ionizing radiations and chemical mutagens. Hereditas 63: 257-278.

Fujii, T., M.Ikenaga and J.T.Lyman (1966) Radiation effects on Arabidopsis thaliana. II. Killing and mutagenic efficiencies of heavy ionizing particles. Radiat. Bot. 6: 297-306.

Gustafsson, A. (1969) A study on induced mutations in plants. In: Induced mutations in plants. Proceedings of a symposium on the nature, induction and utilization of mutations in plants jointly organized by the IAEA and FAO and held at Pullman, Wash., 14-18 July 1969, International Atomic Energy Agency, Vienna, pp.9-31.

Hamatani, M., Y.Iitsuka, T.Abe, K. Miyoshi, M. Yamamoto and S.Yoshida (2001) Mutant flowers of dahlia (Dahlia pinnata Cav.) induced by heavy-ion beams. RIKEN Accel. Prog. Rep. 34: 169.

Hara, Y., T.Abe, K.Sakamoto, Y.Miyazawa and S. Yoshida (2003) Effects of heavy-ion beam irradiation in rose (Rosa Hybrid cv. 'Bridal Fantasy'). RIKEN Accel. Prog. Rep. 36: 135.

Hase, Y., A.Tanaka, T.Baba and H.Watanabe (2000) FRL1 is required for petal and sepal development in Arabidopsis. Plant J. 24: 21-32.

Hirono, Y., H. H.Smith, J.T.Lyman, K.H.Thompson and J.W.Baum (1970) Relative biological effectiveness of heavy ions in producing mutations, tumors, and growth inhibition in the crucifer plant, Arabidopsis. Radiat. Res. 44: 204-223.

Kanaya,T., H.Saito, Y.Hayashi, N.Fukunishi, H.Ryuto, K.Miyazaki, T.Kusumi, T.Abe and K.Suzuki (2008) Heavy-ion beam-induced sterile mutants of verbena (Verbena $\times$ hybrida) with an improved flowering habit. Plant Biotechnol. 25: 91-96.

Kazama,Y., H.Saito, M.Miyagai, H.Takehisa, H.Ichida, Y.Miyazawa, K.Mishiba, T.Kanaya, K.Suzuki, C.H.Bae, K.Miyoshi, M.Mii and T.Abe (2008) Effect of heavy ion-beam irradiation on plant growth and mutation induction in Nicotiana tabacum. Plant Biotechnol. 25: 105-111.

Konzak,C.F., R.A.Nilan, J.Wagner and R.J.Foster (1965) Efficient chemical mutagenesis. In: The use of induced mutations in plant breeding. Report of the FAO/IAEA technical meeting organized by the food and agriculture organization of the United Nations and the International Atomic Energy Agency in cooperation with the European Association for Research on Plant Breeding, Rome, Italy, 25 May 1964, Pergamon Press, Oxford, pp. 49-70.

Matsuo, T., H.Yamaguchi and A.Ando (1958) A comparison of biological effects between thermal neutrons and X-rays on rice seeds. Jpn. J. Breed. 8: 37-45.

Mei, M., H.Deng, Y.Lu, C.Zhuang, Z.Liu, Q.Qiu, Y.Qiu and T.C.Yang (1994) Mutagenic effects of heavy ion radiation in plants. Adv. Space Res. 14: 363-372.

Mikaelsen, K., K.Karunakaran and I.S.Kiss (1971) Mutagenic effectiveness and efficiency of gamma rays, fast neutrons and ethyl methane sulphonate in rice. In: Rice breeding with induced mutations. III. Report of an FAO/IAEA research co-ordination meeting on the use of induced mutations in rice breeding, India, September 1969. Technical Reports Series No. 131. International Atomic Energy Agency, Vienna, pp.91-96.

Miyazaki,K., K.Suzuki, T.Abe, Y.Katsumoto, S.Yoshida and T. Kusumi (2002) Isolation of variegated mutants of Petunia hybrida using heavy-ion beam irradiation. RIKEN Accel. Prog. Rep. 35: 130 .

Miyazaki, K., K.Suzuki, K.Iwaki, T.Kusumi, T.Abe, S.Yoshida and 
H.Fukui (2006) Flower pigment mutations induced by heavy ion beam irradiation in an inter specific hybrid of Torenia. Plant Biotechnol. 23: 163-167.

Nagatomi,S., A.Tanaka, A.Kato, H.Watanabe and S. Tano (1996) Mutation induction through tissue cultures of chrysanthemum by ion beam irradiation. Breed. Sci. 46 (Suppl. 1): 62.

Naito,K., M.Kusaba, N.Shikazono, T.Takano, A.Tanaka, T.Tanisaka and M.Nishimura (2005) Transmissible and nontransmissible mutations induced by irradiating Arabidopsis thaliana pollen with $\gamma$ rays and carbon ions. Genetics 169: 881-889.

Nilan,R.A., C.F.Konzak, J.Wagner and R.R.Legault (1965) Effectiveness and efficiency of radiations for inducing genetic and cytogenetic changes. In: The use of induced mutations in plant breeding. Report of the FAO/IAEA technical meeting organized by the Food and Agriculture Organization of the United Nations and the International Atomic Energy Agency in cooperation with the European Association for Research on Plant Breeding, Rome, Italy, 25 May 1964, Pergamon Press, Oxford, pp. 71-89.

Okamura, M., N. Yasuno, M.Ohtsuka, A. Tanaka, N.Shikazono and Y.Hase (2003) Wide variety of flower-color and -shape mutants regenerated from leaf cultures irradiated with ion beams. Nucl. Instr. and Meth. in Phys. Res. B 206: 574-578.

Osone,K. (1963) Studies on the developmental mechanism of mutated cells induced in irradiated rice seeds. Jpn. J. Breed. 13: 1-13.
Shikazono,N., Y.Yokota, S.Kitamura, C.Suzuki, H.Watanabe, S.Tano and A.Tanaka (2003) Mutation rate and novel tt mutants of Arabidopsis thaliana induced by carbon ions. Genetics 163: 14491455.

Tanaka, A., S. Tano, T.Chantes, Y.Yokota, N.Shikazono and H. Watanabe (1997) A new Arabidopsis mutant induced by ion beams affects flavonoid synthesis with spotted pigmentation in testa. Genes Genet. Syst. 72: 141-148.

Tanaka,A. (1999) Mutation induction by ion beams in Arabidopsis. Gamma Field Symp. 38: 19-27.

Tanaka,A., A.Sakamoto, Y.Ishigaki, O.Nikaido, G.Sun, Y.Hase, N.Shikazono, S.Tano and H.Watanabe (2002) An ultraviolet-Bresistant mutant with enhanced DNA repair in Arabidopsis. Plant Physiol. 129: 64-71.

van Harten, A.M. (1998) Mutation breeding. Cambridge University Press, Cambridge, UK.

Yamaguchi,H. (1962) The chimaeric formation in an $X_{1}$ panicle after irradiation of dormant rice seed. Radiat. Bot. 2: 71-77.

Yamaguchi, H., S. Nagatomi, T.Morishita, K.Degi, A. Tanaka, N. Shikazono and Y.Hase (2003) Mutation induced with ion beam irradiation in rose. Nucl. Instr. Meth. In Phys. Res. B 206: 561-564.

Yang,T.C. and C.A.Tobias (1979) Potential use of heavy-ion radiation in crop improvement. Gamma Field Symp. 18: 141-154. 